\title{
Chemical Variation and Implications on Repellency Activity of Tephrosia vogelii (Hook f.) Essential Oils Against Sitophilus zeamais Motschulsky
}

\author{
Nasifu Kerebba ${ }^{1}\left(\mathbb{D}\right.$, Adebola O. Oyedeji $^{2}{ }^{(}$, Robert Byamukama $^{3}$, Simon K. Kuria $^{4}$ and \\ Opeoluwa O. Oyedeji ${ }^{1, *}$ \\ 1 Department of Chemistry, University of Fort Hare, P/BagX1314, Alice 5700, South Africa; \\ nkerebba@gmail.com \\ 2 Department of Chemical and Physical Sciences, Walter Sisulu University, P/BagX1, Mthatha 5117, \\ South Africa; aoyedeji@gmail.com \\ 3 Department of Chemistry, Makerere University, Kampala P.O. Box 7062, Uganda; \\ rbyamukama@cns.mak.ac.ug \\ 4 Department of Biological and Environmental Sciences, Walter Sisulu University, P/BagX1, Mthatha 5117, \\ South Africa; kkuria@wsu.ac.za \\ * Correspondence: ooyedeji@ufh.ac.za
}

Received: 18 February 2020; Accepted: 26 March 2020; Published: 12 May 2020

\begin{abstract}
The aim of this research is to characterize the variation in the chemical composition of Tephrosia vogelii essential oils from different locations and to investigate the repellency of essential oils against Sitophilus zeamais. Chemical variability in the components of T. vogelii essential oils from eastern Uganda was identified using principal component analysis (PCA) and agglomerative hierarchical clustering (AHC). Based on the profiles of the compounds of the farnesene family, three chemotypes were found: farnesol (chemotype 1$)$, springene ( $\beta$-springene and $\alpha$-springene) and $\beta$-farnesene were all distinctive in chemotype 2 and a mixed variety of farnesol and springene. In the three cases, alkyl benzenes (o-xylene, $\mathrm{m}$-xylene and ethylbenzene) were significant components in the oil. The compounds 1,4-dihydroxy-p-menth-2-ene, 6,10-dimethyl-5,9-undecadien-2-one, and 3,4-dimethyl-3-cyclohexen-1-carboxaldehyde were other prominent constituents. The yields of the essential oils did not vary significantly, however the chemical composition varied with harvesting time during the rainy and dry seasons. In choice repellency tests, chemotype 1 and chemotype 2 were more active against Sitophilus zeamais than the mixed chemotype. Farnesol was found to be effective only at a higher concentration as a repellent against $S$. zeamais. We therefore hypothesize that farnesol is a key player in this and we demonstrated the weak repellency of this compound. However, further study that aims to optimize and standardize the varieties and harvesting period is needed for recommendation to smallhold farmers.
\end{abstract}

Keywords: chemotypes; Tephrosia vogelii; repellency activity; Sitophilus zeamais; essential oils

\section{Introduction}

Tephrosia vogelii (Hook f.) is a pesticidal plant, found mainly in the tropics in Africa [1]. It is also called fish poison [2]. It is a soft woody branching herb with dense foliage and can grow up to $0.5-4.0 \mathrm{~m}$ tall [3]. It occurs in climates with an annual rainfall of $850-2650 \mathrm{~mm}$ and an annual mean temperature of $12.5-26.2^{\circ} \mathrm{C}$, and is found up to $2100 \mathrm{~m}$ above sea level [3]. Attempts have been made in eastern and southern Africa to promote T. vogelii for wider application as a pesticide source through earlier reports [4,5]. Investigations of crude plant extracts and methanol/hexane extracts protect stored maize against the weevil S. zeamais Motschulsky (Coleoptera: Curculionidae) and more basic mortality 
studies without chemistry elements have previously been done [6,7]. Some reports however indicate that some plants like T. vogelii and Lippia javanica (Burm. f.) can exhibit an extreme variation of bioactive principles from the same species [8,9]. The work presented in [8] showed that some crude extracts of the leaves of $T$. vogelii possessed rotenoids and were thus pesticidal materials (chemotype 1), while others were non-pesticidal due to lack of rotenoids (chemotype 2). This variation was thus based on the profiles of the flavonoids. Additionally, recent reports have identified three chemotypes of T. vogelii materials from east Africa (Kenya and Tanzania) and Malawi through phytochemical analysis [10]. These variations in the phytocompounds would affect the pesticidal activities of these plants, pausing limitation to their use which would slow their adoption.

The novelty of this study comes largely from the fact that in this case, the essential oil is used rather than a solvent extract. Some studies of T. vogelii essential oil composition have also been carried out previously [11,12], but the quality of these studies may be questionable. It should be noted that some of the chemical components have already been individually tested against Sitophilus species (most often S. oryzae) for repellency, mortality or both in other studies [13]; for farnesol, $\alpha$-pinene in [14]; while ethylbenzene has been reported to be released from Sitophilus-infested grain. To the best of our knowledge, chemical variation in the essential oils of T. vogelii species and the implications in pest control does not exist. This paper seeks to characterize the variation in chemical composition of T. vogelii essential oil from different locations over the course of three years. It then investigates the repellency of $T$. vogelii essential oil against $S$. zeamais.

\section{Materials and Methods}

\subsection{Plant Collection and Sites}

Collection of plant materials took place in the Butaleja district, eastern Uganda. Two plant collection sites were considered for the study: Mazimasa sub-county, Nampologoma Parish, Muyago village and Kachonga sub-county, Kyadongo parish, and Kyadongo B village. Kachonga sub-county surrounds the Doho Wetland found in Mazimasa sub-county and the area is ten kilometers from Mbale Town $\left(33^{\circ} 55^{\prime}\right.$ to $34^{\circ} 05^{\prime} \mathrm{E}$ and $0^{\circ} 50^{\prime}$ to $\left.1^{\circ} 00^{\prime} \mathrm{N}\right)$. The coordinates of the district are: $00^{\circ} 56^{\prime} \mathrm{N}, 33^{\circ} 57^{\prime} \mathrm{E}$. The Butaleja district area is approximately $653.1 \mathrm{~km}^{2}$. The altitude of the district ranges from $1050 \mathrm{~m}$ to $1100 \mathrm{~m}$ above sea level. Several tropical climate conditions with average temperatures between $16^{\circ} \mathrm{C}$ and $29^{\circ} \mathrm{C}$ occur due to different altitudes. The mean annual rainfall varies between $1500 \mathrm{~mm}$ to $1750 \mathrm{~mm}$ and is received within four months [15]. The bimodal rainfall peaks are March-May and August-September [16]. The soil is sandy with low organic content, although some clay soils transferred from the neighboring volcanic mountain in Mbale district form along rivers [15].

\subsection{Plant Materials and Botanical Identification}

Different plant leaf materials of T. vogelii plant species were collected from Muyago and Kyadongo villages, Butaleja District, eastern Uganda. Leafy materials were collected from branches, air dried and stored. To determine the effect of geographical and seasonal variations in the existence of $T$. vogelii chemotypes, the collection of plant materials was done within two major seasonal rainfall patterns in the district: rainy season (March-May, and August-September) and dry season (January and June-July) (Table 1) from two different villages.

The distance between Muyago and Kyadongo villages is about $7 \mathrm{~km}$. The collected plant species were identified by a senior botanist, Rwaburindori Protase, at the Department of Botany, Makerere University. The voucher specimen was deposited at Makerere University Herbarium and the voucher name, number (Kerebba N. No 1-Tephrosia vogelii Hook. f. (Leguminosae) (Access No. MHU 50735)) and GPS coordinates were deposited at the Herbarium. 
Table 1. Location of sampling points.

\begin{tabular}{|c|c|c|c|c|c|}
\hline \multirow{2}{*}{ Village (Sample) } & \multirow{2}{*}{ Flower Color } & \multicolumn{2}{|c|}{ Location } & \multirow{2}{*}{ Sampling Date } & \multirow{2}{*}{$\begin{array}{l}\text { Altitud } \\
(\mathrm{m})\end{array}$} \\
\hline & & Latitude North & Longitude East & & \\
\hline Muyago (TV1 muya) & White & $0^{\circ} 84^{\prime} 20^{\prime \prime}$ & $34^{\circ} 03^{\prime} 15^{\prime \prime}$ & 14 May 2017 & 1080 \\
\hline KyadongoB (TV1 kya) & White & $0^{\circ} 90^{\prime} 14^{\prime \prime}$ & $34^{\circ} 08^{\prime} 30^{\prime \prime}$ & 1 June 2017 & 1098 \\
\hline Kyadongo B (TV1 kyb) & White & $0^{\circ} 90^{\prime} 30^{\prime \prime}$ & $34^{\circ} 09^{\prime} 45^{\prime \prime}$ & 1 June 2017 & 1098 \\
\hline Kyadongo B (TV1 kyc) & White & $0^{\circ} 80^{\prime} 10^{\prime \prime}$ & $34^{\circ} 08^{\prime} 40^{\prime \prime}$ & 1 June 2017 & 1098 \\
\hline Muyago (TV2 muya) & White & $0^{\circ} 84^{\prime} 14.9^{\prime \prime}$ & $34^{\circ} 03^{\prime} 10^{\prime \prime}$ & 15 August 2017 & 1080 \\
\hline Kyadongo B (TV2 kya) & White & $0^{\circ} 70^{\prime} 30^{\prime \prime}$ & $34^{\circ} 07^{\prime} 35^{\prime \prime}$ & 15 August 2017 & 1098 \\
\hline Kyadongo B (TV2 kyb) & White & $0^{\circ} 90^{\prime} 14^{\prime \prime}$ & $34^{\circ} 08^{\prime} 30^{\prime \prime}$ & 15 August 2017 & 1098 \\
\hline Kyadongo B (TV2 kyc) & White & $0^{\circ} 90^{\prime} 20^{\prime \prime}$ & $34^{\circ} 09^{\prime} 40^{\prime \prime}$ & 15 August 2017 & 1098 \\
\hline Kyadongo B (TV2 kyd) & White & $0^{\circ} 90^{\prime} 35^{\prime \prime}$ & $34^{\circ} 08^{\prime} 45^{\prime \prime}$ & 15 August 2017 & 1098 \\
\hline Muyago (TV3 muya) & White & $0^{\circ} 84^{\prime} 00^{\prime \prime}$ & $34^{\circ} 02^{\prime} 45^{\prime \prime}$ & 1 March 2018 & 1090 \\
\hline Muyago (TV3 muyb) & White & $0^{\circ} 84^{\prime} 14^{\prime \prime}$ & $34^{\circ} 03^{\prime} 09^{\prime \prime}$ & 1 March 2018 & 1090 \\
\hline Muyago (TV3 muyc) & White & $0^{\circ} 84^{\prime} 14.9^{\prime \prime}$ & $34^{\circ} 03^{\prime} 10^{\prime \prime}$ & 1 March 2018 & 1090 \\
\hline Kyadongo B (TV3 kya) & White & $0^{\circ} 90^{\prime} 14^{\prime \prime}$ & $34^{\circ} 08^{\prime} 30^{\prime \prime}$ & 1 March 2018 & 1098 \\
\hline Kyadongo B (TV4 kya) & White & $0^{\circ} 70^{\prime} 30^{\prime \prime}$ & $34^{\circ} 07^{\prime} 35^{\prime \prime}$ & 10 January 2019 & 1098 \\
\hline Kyadongo B (TV4 kyb) & White & $0^{\circ} 90^{\prime} 14^{\prime \prime}$ & $34^{\circ} 08^{\prime} 30^{\prime \prime}$ & 10 January 2019 & 1098 \\
\hline Kyadongo B (TV4 kyc) & White & $0^{\circ} 90^{\prime} 20^{\prime \prime}$ & $34^{\circ} 09^{\prime} 40^{\prime \prime}$ & 10 January 2019 & 1098 \\
\hline Muyago (TV4 muya) & White & $0^{\circ} 84^{\prime} 00^{\prime \prime}$ & $34^{\circ} 02^{\prime} 45^{\prime \prime}$ & 10 January 2019 & 1090 \\
\hline Muyago (TV4 muyb) & White & $0^{\circ} 84^{\prime} 14^{\prime \prime}$ & $34^{\circ} 03^{\prime} 09^{\prime \prime}$ & 10 January 2019 & 1090 \\
\hline Muyago (TV4 muyc) & White & $0^{\circ} 84^{\prime} 14.9^{\prime \prime}$ & $34^{\circ} 03^{\prime} 10^{\prime \prime}$ & 10 January 2019 & 1090 \\
\hline
\end{tabular}

Rain season sampling was during March, May and August while dry season sampling was during January and June. Muy $=$ Samples collected from Muyago, $\mathrm{Ky}=$ Samples collected from Kyadongo and TV = Tephrosia vogelii . a, b, c and d; signify different samples while numbers 1, 2, 3, and 4 depict sampling period.

\subsection{Extraction and Analysis of Essential Oils}

\subsubsection{Extraction}

Each sample of plant leaf materials (20 g) was hydro-distilled for $4 \mathrm{~h}$ using a Clevenger apparatus set up as prescribed by British pharmacotia for essential oils. The oils were collected using a Pasteur pipette and dried using anhydrous sodium sulphate. The dry oil was then put in a small weighed dark brown bottle $(5 \mathrm{~mL})$ and refrigerated at $4{ }^{\circ} \mathrm{C}$ for analysis. For repellency evaluation, more masses of the sample were hydro-distilled.

\subsubsection{Identification and Quantification of Compounds}

Synthetic Chemicals

Ethylbenzene $(>99.8 \%),( \pm)$-linalool $(>95.0 \%)$, 2-undecanone (95\%), o-xylene $(\geq 99.0 \%)$, p-cymene $(>95.0 \%)$ and R-(+)-limonene $(>98.0 \%)$ and undecanoic acid $(>99.0 \%)$ were purchased from Sigma-Aldrich (Gillingham, Dorset, UK). n-Decane ( $>99.0 \%)$ was bought from British Drug Houses $(\mathrm{BDH})$ chemicals. $\alpha$-Terpeneol $(98.0 \%)$ was purchased from Fisher Scientific (Loughborough, Leicestershire, UK). A mixture of xylene isomers (o-xylene and m-xylene) was purchased from pronalys while $\alpha$-pinene was purchased from B.C. Treatt \& Co. Ltd. (E)- $\beta$-farnesene $(\geq 98 \%)$ and trans-(2E,6E)-farnesol $(98.0 \%)$ standards were purchased from career henan chemical co., China.

Identification of Compounds with Gas Chromatography (GC)

GC analysis was done using Brunker 300 Gas Chromatograph equipped with A flame ionization detector (FID) detector and ZB-5 column (30 $\mathrm{m}$ in length $\times 0.25 \mathrm{~mm}$ i.d. $\times 0.25 \mu \mathrm{m}$ film thickness). The carrier gas was hydrogen at a flow rate of $1.0 \mathrm{~mL} / \mathrm{min}$ and inlet pressure of $52.6 \mathrm{KPa}$. The column oven temperature was programmed to $50-250{ }^{\circ} \mathrm{C}$ at a rate of $3.0^{\circ} \mathrm{C} / \mathrm{min}$. Injector and detector temperature were set at $250^{\circ} \mathrm{C}$, the volume injector was set to $1.0 \mu \mathrm{L}$ of the oil, and the split ratio was 1:5. Peaks were measured by electronic integration. n-Alkanes of $C_{8}$ to $C_{30}$ were run under the same condition for Kovats indices determination [17]. 
Identification and Quantification of Volatile Constituents by Gas Chromatography-Mass Spectrometry (GC/MS)

The essential oil was analyzed by a Bruker 300-MS along with the 431-GC and CP-8400 autosampler (quadrupole mass spectrometer) equipped with a ZB-5 capillary column (30 m length $\times 0.25 \mathrm{~mm}$ i.d. $\times 0.25 \mu \mathrm{m}$ film thickness). The oven temperature was programmed from $50{ }^{\circ} \mathrm{C}-250{ }^{\circ} \mathrm{C}$ at a rate of $3.0^{\circ} \mathrm{C} / \mathrm{min}$, electron ionization was at $70 \mathrm{eV}$. Helium was used as the carrier gas at a flow rate of $1.0 \mathrm{~mL} / \mathrm{min}$. Injector and detector temperature were set at $280^{\circ} \mathrm{C}$; the split ratio was $1: 5$. One microliter of the diluted oil in hexane was injected into the GC/MS. Compounds in the essential oil were identified by matching their Kovats indices and mass spectra with the ones recorded in WILEY NIST 11 library and by comparing them with literature values [18]. Where possible, authentic compounds were co-injected. To quantify the amount for constituents in the oil, standard solutions of 5, 10, 20, 40 and between $70 \leq \mathrm{Y} \leq 100 \mathrm{ppb}$, ( $\mathrm{Y}$, was the equivalent of a 5 or $10 \mu \mathrm{L}$ stock of the compound used given that the densities of the standards were different) for the linear regression curves were prepared from synthetic reference materials. These were run on the same day as the sample analysis and regression equations were obtained by plotting peak areas against the concentration levels. For compounds whose standards were available, quantification was done. For unavailable standards, compounds were grouped into chemical classes (hydrocarbons, alkylbenzenes, aldehydes, alcohols, etc.) and subclasses (monoterpenes, sesquiterpenes, oxygenated monoterpenes etc.) and a semi-quantification approach was carried out using one (or more) reference standard per group. The compound composition was then expressed as percentage peak area, i.e.,:

$$
\text { Constituent percentage peak area }=(\mathrm{Xs}) \times 100 /(1000 \times \mathrm{R})
$$

where $\mathrm{Xs}_{\mathrm{s}}$ is the constituent concentration with respect to its peak area $\left(\mathrm{ppb} / \mu \mathrm{g} \mathrm{mL} \mathrm{m}^{-1}\right)$ relative to peak area in the injection volume $(1 \mu \mathrm{L}=1000 \mathrm{ppb})$, and $\mathrm{R}$ is the recovery ( $\mathrm{R}$ was taken as $100 \%$ since average recovery on spiking was $93.1 \pm 9.8, n=11$ ).

\subsection{Principal Component Analysis (PCA) and Agglomerative Hierarchical Clustering (AHC) of Major Chemical Components from Oils of T. vogelii Species}

Principal component analysis (PCA) and agglomerative hierarchical clustering (AHC) were performed on the data to group components and samples into clusters using statistical software SPSS for Windows version 25. PCA is a statistical tool that aims to represent the variation present in the data. It allows similarities and differences between data to be seen easily. During PCA, values in the loadings matrix were obtained through the transformation of data from correlated to new uncorrelated variables called principal components [19]. PCA was performed on a combined set of data from the two locations, giving 19 samples $\times 23$ variables for PCA. Analysis followed the standardization of data using Varimax rotation. Factor loadings generated indicate the correlations of each chemical constituent with its corresponding component. Loading scores which were greater than $5 \%$ of the variance of a given variable were considered, however only loadings higher than an absolute value of 0.23 were considered meaningful throughout the analysis.

$\mathrm{AHC}$ is an algorithm that brings together related objects into clusters. The clustering makes it easier to see the correlations. The endpoint is having clusters that are distinctive from other clusters and the objects within a cluster are very similar. AHC, based on Euclidean distance, was used to analyze seasonal and geographical influence on the yield and composition of the samples of T. vogelii. Finally, the classification of samples was done based on the composition and chemical constituents.

\subsection{Repellency Evaluation}

The repellency of the chemotypes was evaluated for selected samples (Tv1kyb, Tv4kyc and TV4muyc). 


\subsubsection{Rearing of Weevils}

Plastic containers were used to breed colonies of S. zeamais. An initial stock of maize weevils was obtained from infected maize from a market in Mthatha, Eastern Cape province of South Africa. Weevils were identified by Simon Kuria, an entomologist, of the Department of Biological and Environment Sciences, Walter Sisulu University. Culturing occurred at $25-29^{\circ} \mathrm{C}, 60 \pm 5 \%$ relative humidity (RH) and a photoperiod of 12:12 dark:light.

\subsubsection{Repellence Bioassay against S. zeamais}

Repellence assays were performed using the area preference method [20]. Here, glass Petri dishes $(9.0 \mathrm{~cm} \times 1.2 \mathrm{~cm})$ and half discs of filter paper $\left(31.8 \mathrm{~cm}^{2}\right)$ were used. Different levels of the test solutions $\left(1 \mu \mathrm{L} / \mathrm{mL}, 5 \mu \mathrm{L} / \mathrm{mL}\right.$ and $10 \mu \mathrm{L} / \mathrm{mL}$ of essential oils corresponding to $0.03 \mu \mathrm{L}$ of oil per $\mathrm{cm}^{3}, 0.16 \mu \mathrm{L}$ of oil per $\mathrm{cm}^{3}$ and $0.31 \mu \mathrm{L}$ of oil per $\mathrm{cm}^{3}$, respectively) were used to check for the repellent potential of essential oils. Whatman filter paper discs were cut into two halves. The oil treatment was applied to one half uniformly using a micropipette. The other half was a control treatment of $1.0 \mathrm{~mL}$ of hexane. Both treated halves were allowed to dry so that the solvent could evaporate completely. The halves were then attached with cellophane tape in a manner that would avoid the seepage of the test samples from one disc to another and placed at the bottom of each petri dish. Thirty mixed sex-adult S. zeamais were released at the center of each disc and the petri dish was covered and kept in the dark at $25-29.5^{\circ} \mathrm{C}$. Three replicates were done for each test solution of the essential oil simultaneously. The numbers of the weevils in both the treated and untreated filter paper discs were counted after $1 \mathrm{~h}, 12 \mathrm{~h}, 24 \mathrm{~h}, 48 \mathrm{~h}$ and $72 \mathrm{~h}$.

Percentage repellency (PR) was calculated using the formula in Equation (1):

$$
P R(\%)=\frac{C-T}{C+T}
$$

$C=$ insect number found on untreated half,

$T=$ insect found on treated half.

Preference index (PI) was obtained using the formula in Equation (2):

$$
P I=\frac{A-B}{A+B}
$$

$A=$ percentage of insects in treated halves,

$B=$ percentage of insects in untreated halves.

The experiments were successively repeated twice, with three and two replicates each time, and separate controls were set in all the replicates in a completely randomized design and non-random design, respectively. Data were treated as mean percentage repellency \pm standard error of the mean (SEM) (see Supplementary Materials).

\section{Results and Discussion}

\subsection{Chemical Constituents and Composition of Essential Oils}

Yellow distillates whose percentage yield ranged between $0.18 \pm 0.01 \%$ to $0.22 \pm 0.01 \%(w / w)$ dry weight for samples from Kyadongho B (Table 2) and $0.16 \pm 0.00 \%$ to $0.22 \pm 0.01 \%(w / w)$ dry weight for Muyago samples (Table 3) were obtained from the hydro distillation of leaf materials. 
Table 2. Mean peak area ( $\% \pm$ standard error of the mean (SEM)) composition of major chemical constituents identified in the essential oils of T. vogelii samples from Kyadogho B.

\begin{tabular}{|c|c|c|c|c|c|c|c|c|c|c|c|c|}
\hline $\begin{array}{c}\text { Compounds } \\
(\mathrm{RT}, \mathrm{KI})^{1}\end{array}$ & $\begin{array}{l}\text { TV1 } \\
\text { kya }\end{array}$ & $\begin{array}{l}\text { TV1 } \\
\text { kyb }\end{array}$ & $\begin{array}{l}\text { TV1 } \\
\text { kyc }\end{array}$ & $\begin{array}{l}\text { TV2 } \\
\text { kya }\end{array}$ & $\begin{array}{l}\text { TV2 } \\
\text { kyb }\end{array}$ & $\begin{array}{l}\text { TV2 } \\
\text { kyc }\end{array}$ & $\begin{array}{l}\text { TV2 } \\
\text { kyd }\end{array}$ & $\begin{array}{l}\text { TV3 } \\
\text { kya }\end{array}$ & $\begin{array}{l}\text { TV4 } \\
\text { kya }\end{array}$ & $\begin{array}{l}\text { TV4 } \\
\text { kyb }\end{array}$ & $\begin{array}{l}\text { TV4 } \\
\text { kyc }\end{array}$ & $\begin{array}{c}\text { Identification } \\
\text { Method }^{2}\end{array}$ \\
\hline $\begin{array}{c}\text { Ethylbenzene } \\
(4.753,878)\end{array}$ & $\begin{array}{c}0.2 \\
(0.0)\end{array}$ & $\begin{array}{c}4.0 \\
(0.4)\end{array}$ & $\begin{array}{c}0.6 \\
(0.3)\end{array}$ & $\begin{array}{c}0.4 \\
(0.1)\end{array}$ & $\begin{array}{c}1.4 \\
(0.4)\end{array}$ & $\begin{array}{c}0.2 \\
(0.0)\end{array}$ & $\begin{array}{c}0.5 \\
(0.0)\end{array}$ & $\begin{array}{c}0.7 \\
(0.1)\end{array}$ & nd & nd & nd & $\begin{array}{l}\mathrm{MS} / \mathrm{KI} / \\
\mathrm{CI}\end{array}$ \\
\hline $\begin{array}{c}\text { o-Xylene } \\
(4.962,867)\end{array}$ & $\begin{array}{c}1.1 \\
(0.0)\end{array}$ & $\begin{array}{l}29.4 \\
(1.6)\end{array}$ & $\begin{array}{c}3.2 \\
(2.1)\end{array}$ & $\begin{array}{c}2.1 \\
(0.3)\end{array}$ & $\begin{array}{c}9.0 \\
(1.7)\end{array}$ & $\begin{array}{l}1.1 \\
(0.1)\end{array}$ & $\begin{array}{c}3.4 \\
(0.6)\end{array}$ & $\begin{array}{c}6.7 \\
(2.3)\end{array}$ & nd & nd & nd & $\begin{array}{l}\mathrm{MS} / \mathrm{KI} / \\
\mathrm{CI}\end{array}$ \\
\hline $\begin{array}{l}\text { p-Xylene } \\
(5.448,887)\end{array}$ & $\begin{array}{c}0.9 \\
(0.0)\end{array}$ & nd & $\begin{array}{l}1.6 \\
(0.6)\end{array}$ & nd & nd & nd & $\begin{array}{c}1.5 \\
(0.1)\end{array}$ & nd & nd & nd & nd & $\mathrm{MS} / \mathrm{KI}$ \\
\hline $\begin{array}{c}\text { m-Xylene } \\
(5.539,896)\end{array}$ & $\begin{array}{c}0.3 \\
(0.0)\end{array}$ & $\begin{array}{l}25.0 \\
(1.2)\end{array}$ & $\begin{array}{c}3.6 \\
(1.6)\end{array}$ & $\begin{array}{c}1.0 \\
(0.0)\end{array}$ & $\begin{array}{c}5.1 \\
(0.8)\end{array}$ & $\begin{array}{c}0.3 \\
(0.0)\end{array}$ & $\begin{array}{c}2.1 \\
(0.1)\end{array}$ & $\begin{array}{c}3.3 \\
(0.6)\end{array}$ & nd & nd & nd & $\begin{array}{l}\mathrm{MS} / \mathrm{KI} / \\
\mathrm{CI}\end{array}$ \\
\hline $\begin{array}{c}\text { D-(+)-Alpha-pinene } \\
(6.811,931)\end{array}$ & $\begin{array}{c}0.7 \\
(0.1)\end{array}$ & $\begin{array}{l}1.7 \\
(0.1)\end{array}$ & $\begin{array}{c}0.7 \\
(0.1)\end{array}$ & $\begin{array}{c}0.5 \\
(0.1)\end{array}$ & $\begin{array}{l}1.0 \\
(0.2)\end{array}$ & nd & $\begin{array}{c}0.5 \\
(0.1)\end{array}$ & nd & $\begin{array}{c}0.4 \\
(0.1)\end{array}$ & $\begin{array}{c}0.9 \\
(0.2)\end{array}$ & nd & $\begin{array}{l}\mathrm{MS} / \mathrm{KI} / \\
\mathrm{CI}\end{array}$ \\
\hline $\begin{array}{c}\text { D-Limonene } \\
(10.419,1031)\end{array}$ & nd & $\begin{array}{c}0.2 \\
(0.0)\end{array}$ & $\begin{array}{c}0.2 \\
(0.0)\end{array}$ & $\begin{array}{c}0.8 \\
(0.4)\end{array}$ & $\begin{array}{c}0.2 \\
(0.0)\end{array}$ & nd & $\begin{array}{c}0.1 \\
(0.0)\end{array}$ & nd & nd & $\begin{array}{c}0.1 \\
(0.0)\end{array}$ & nd & $\begin{array}{l}\mathrm{MS} / \mathrm{KI} / \\
\mathrm{CI}\end{array}$ \\
\hline $\begin{array}{c}\text { Linalool } \\
(13.514,1102)\end{array}$ & nd & $\begin{array}{c}1.8 \\
(0.0)\end{array}$ & nd & nd & nd & nd & nd & nd & nd & nd & nd & $\begin{array}{l}\mathrm{MS} / \mathrm{KI} / \\
\mathrm{CI}\end{array}$ \\
\hline $\begin{array}{c}(\mathrm{E}, \mathrm{E}) \text {-Cosmene } \\
(15.875,1132)\end{array}$ & nd & nd & nd & nd & nd & nd & nd & nd & $\begin{array}{c}0.2 \\
(0.1)\end{array}$ & $\begin{array}{c}0.6 \\
(0.1)\end{array}$ & $\begin{array}{c}0.5 \\
(0.0)\end{array}$ & $\mathrm{MS} / \mathrm{KI}$ \\
\hline $\begin{array}{l}\text { Isocaryophyllene } \\
(30.511,1409)\end{array}$ & nd & nd & nd & nd & nd & nd & nd & nd & nd & nd & $\begin{array}{c}0.2 \\
(0.0)\end{array}$ & $\mathrm{MS} / \mathrm{KI}$ \\
\hline $\begin{array}{l}\text { (E)-Nerolidol } \\
(33.432,1564)\end{array}$ & nd & $\begin{array}{c}0.2 \\
(0.0)\end{array}$ & nd & nd & $\begin{array}{c}0.2 \\
(0.0)\end{array}$ & $\begin{array}{c}0.2 \\
(0.0)\end{array}$ & nd & nd & & nd & & $\mathrm{MS} / \mathrm{KI}$ \\
\hline $\begin{array}{l}\text { (-)-Spathulenol } \\
(33.959,1566)\end{array}$ & nd & $\begin{array}{c}0.2 \\
(0.0)\end{array}$ & $\begin{array}{c}0.2 \\
(0.0)\end{array}$ & $\begin{array}{c}0.3 \\
(0.0)\end{array}$ & $\begin{array}{c}0.3 \\
(0.0)\end{array}$ & $\begin{array}{c}0.2 \\
(0.0)\end{array}$ & nd & nd & $\begin{array}{c}0.2 \\
(0.0)\end{array}$ & nd & $\begin{array}{c}0.2 \\
(0.0)\end{array}$ & $\mathrm{MS} / \mathrm{KI}$ \\
\hline $\begin{array}{l}\text { 3,4-Dimethyl-3-cyclohexen-1-carboxaldehyde } \\
(34.520,1492)\end{array}$ & nd & nd & nd & nd & $\begin{array}{c}2.9 \\
(0.6)\end{array}$ & nd & nd & nd & nd & nd & nd & $\mathrm{MS} / \mathrm{KI}$ \\
\hline $\begin{array}{l}\text { Cis-p-metha-1(7)-8-dien-2-ol } \\
(35.208,1233)\end{array}$ & nd & nd & nd & nd & nd & nd & nd & nd & nd & nd & $\begin{array}{c}1.2 \\
(0.0)\end{array}$ & $\mathrm{MS} / \mathrm{KI}$ \\
\hline $\begin{array}{l}\text { Isoaromadendrene epoxide } \\
(37.465,1577)\end{array}$ & nd & $\begin{array}{c}0.2 \\
(0.0)\end{array}$ & nd & nd & nd & nd & nd & $\begin{array}{c}0.2 \\
(0.0)\end{array}$ & $\begin{array}{c}0.3 \\
(0.0)\end{array}$ & $\begin{array}{c}0.3 \\
(0.0)\end{array}$ & $\begin{array}{c}0.7 \\
(0.3)\end{array}$ & $\mathrm{MS} / \mathrm{KI}$ \\
\hline $\begin{array}{c}\text { 1,4-Dihydroxy-p-menth-2-ene } \\
(37.767,1243)\end{array}$ & nd & $\begin{array}{c}0.2 \\
(0.0)\end{array}$ & $\begin{array}{c}0.4 \\
(0.0)\end{array}$ & nd & $\begin{array}{c}1.4 \\
(0.2)\end{array}$ & nd & nd & nd & nd & $\begin{array}{c}1.1 \\
(0.2)\end{array}$ & $\begin{array}{c}0.9 \\
(0.1)\end{array}$ & $\mathrm{MS} / \mathrm{KI}$ \\
\hline
\end{tabular}


Table 2. Cont.

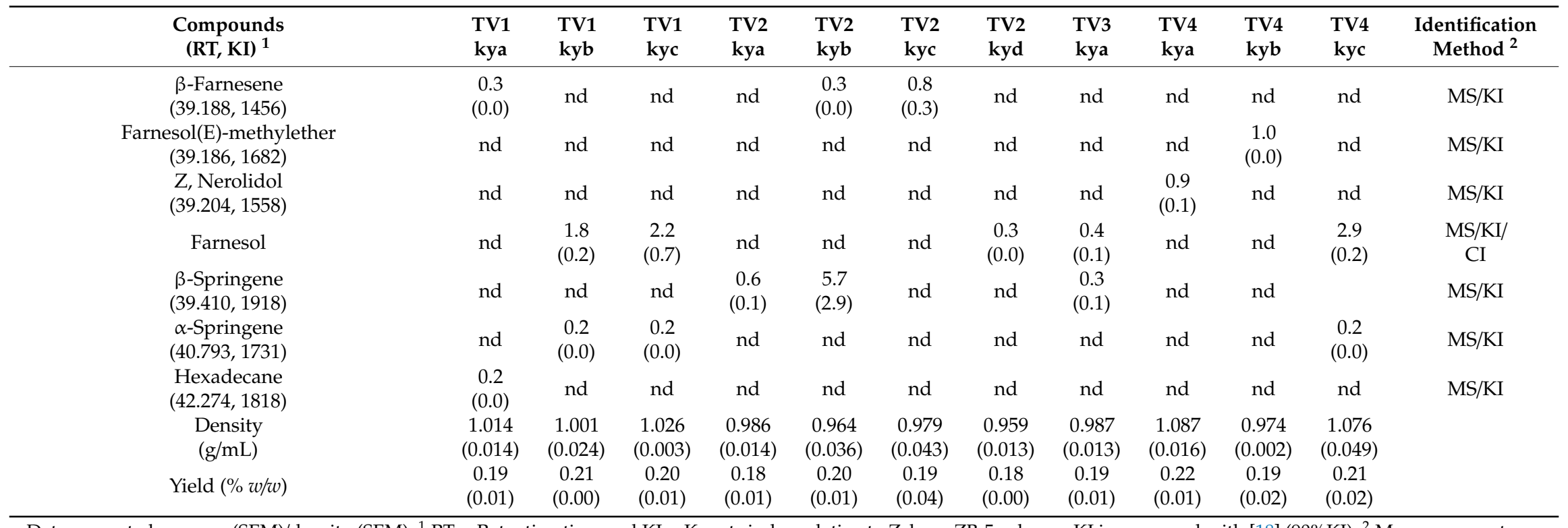

Data presented as mean (SEM)/density (SEM). ${ }^{1} \mathrm{RT}=$ Retention time and KI = Kovats index relative to Zebron ZB-5 column. KI is compared with [18] $(90 \% \mathrm{KI}) .{ }^{2} \mathrm{M}=\mathrm{mass}$ spectrum matching with National Institute of Standards and Technology (NIST) library [17], CI = co-injection with standard.

Table 3. Mean peak area ( $\% \pm$ SEM) composition of major chemical constituents identified in the essential oils of T. vogelii samples from Muyago village.

\begin{tabular}{|c|c|c|c|c|c|c|c|c|c|}
\hline $\begin{array}{c}\text { Compounds } \\
(\mathrm{RT}, \mathrm{KI})^{1}\end{array}$ & $\begin{array}{c}\text { TV1 } \\
\text { muya }\end{array}$ & $\begin{array}{c}\text { TV2 } \\
\text { muya }\end{array}$ & $\begin{array}{l}\text { TV3 } \\
\text { muya }\end{array}$ & $\begin{array}{l}\text { TV3 } \\
\text { muyb }\end{array}$ & $\begin{array}{l}\text { TV3 } \\
\text { muyc }\end{array}$ & $\begin{array}{l}\text { TV4 } \\
\text { muya }\end{array}$ & $\begin{array}{c}\text { TV4 } \\
\text { muyb }\end{array}$ & $\begin{array}{c}\text { Tv4 } \\
\text { muyc }\end{array}$ & $\begin{array}{l}\text { Identification } \\
\text { Method }^{2}\end{array}$ \\
\hline $\begin{array}{c}\text { Ethylbenzene } \\
(4.753,878)\end{array}$ & $\begin{array}{c}2.7 \\
(0.2)\end{array}$ & $\begin{array}{c}2.4 \\
(0.4)\end{array}$ & nd & $\begin{array}{c}1.7 \\
(0.3)\end{array}$ & $\begin{array}{c}2.3 \\
(1.6)\end{array}$ & nd & nd & nd & MS/KI/CI \\
\hline $\begin{array}{c}\text { o-Xylene } \\
(4.962,867)\end{array}$ & $\begin{array}{l}17.6 \\
(0.6)\end{array}$ & $\begin{array}{l}22.1 \\
(1.2)\end{array}$ & $\begin{array}{c}0.9 \\
(0.0)\end{array}$ & $\begin{array}{c}8.6 \\
(0.8)\end{array}$ & $\begin{array}{c}23.4 \\
(17.3)\end{array}$ & nd & nd & nd & MS/KI/CI \\
\hline $\begin{array}{c}\text { p-Xylene } \\
(5.448,887)\end{array}$ & nd & nd & $\begin{array}{c}0.9 \\
(0.0)\end{array}$ & $\begin{array}{c}3.1 \\
(2.0)\end{array}$ & $\begin{array}{c}5.2 \\
(3.4)\end{array}$ & nd & nd & nd & MS/KI \\
\hline $\begin{array}{l}\text { m-Xylene } \\
(5.539,896)\end{array}$ & $\begin{array}{l}14.4 \\
(0.2)\end{array}$ & $\begin{array}{l}18.1 \\
(2.7)\end{array}$ & nd & $\begin{array}{l}5.3 \\
(0.6)\end{array}$ & $\begin{array}{c}6.7 \\
(5.1)\end{array}$ & nd & nd & nd & MS/KI/CI \\
\hline
\end{tabular}


Table 3. Cont.

\begin{tabular}{|c|c|c|c|c|c|c|c|c|c|}
\hline $\begin{array}{c}\text { Compounds } \\
\text { (RT, KI) }{ }^{1}\end{array}$ & $\begin{array}{c}\text { TV1 } \\
\text { muya }\end{array}$ & $\begin{array}{c}\text { TV2 } \\
\text { muya }\end{array}$ & $\begin{array}{c}\text { TV3 } \\
\text { muya }\end{array}$ & $\begin{array}{c}\mathrm{TV3} \\
\text { muyb }\end{array}$ & $\begin{array}{l}\text { TV3 } \\
\text { muyc }\end{array}$ & $\begin{array}{c}\text { TV4 } \\
\text { muya }\end{array}$ & $\begin{array}{c}\text { TV4 } \\
\text { muyb }\end{array}$ & $\begin{array}{c}\text { Tv4 } \\
\text { muyc }\end{array}$ & $\begin{array}{c}\text { Identification } \\
\text { Method }^{2}\end{array}$ \\
\hline $\begin{array}{c}\text { 2-Butoxyethanol } \\
(5.776,895)\end{array}$ & nd & $\begin{array}{c}2.2 \\
(0.7)\end{array}$ & $\begin{array}{c}0.3 \\
(0.1)\end{array}$ & $\begin{array}{c}3.7 \\
(0.9)\end{array}$ & $\begin{array}{c}2.7 \\
(0.1)\end{array}$ & nd & nd & nd & $\mathrm{MS} / \mathrm{KI}$ \\
\hline $\begin{array}{c}\text { D-(+)-Alpha-pinene } \\
(6.811,931)\end{array}$ & $\begin{array}{c}1.2 \\
(0.0)\end{array}$ & $\begin{array}{c}1.6 \\
(0.3)\end{array}$ & nd & $\begin{array}{l}1.5 \\
(0.2)\end{array}$ & $\begin{array}{c}1.8 \\
(1.2)\end{array}$ & $\begin{array}{c}0.4 \\
(0.0)\end{array}$ & $\begin{array}{c}0.6 \\
(0.0)\end{array}$ & nd & $\mathrm{MS} / \mathrm{KI} / \mathrm{CI}$ \\
\hline $\begin{array}{l}\text { D-Limonene } \\
(10.419,1031)\end{array}$ & $\begin{array}{c}0.2 \\
(0.0)\end{array}$ & $\begin{array}{c}0.3 \\
(0.0)\end{array}$ & nd & $\begin{array}{c}0.2 \\
(0.0)\end{array}$ & $\begin{array}{c}0.1 \\
(0.0)\end{array}$ & nd & nd & nd & $\mathrm{MS} / \mathrm{KI} / \mathrm{CI}$ \\
\hline $\begin{array}{c}\text { Linalool } \\
(13.514,1102)\end{array}$ & nd & $\begin{array}{l}1.5 \\
(0.3)\end{array}$ & nd & nd & nd & $\begin{array}{c}1.0 \\
(0.2)\end{array}$ & nd & nd & $\mathrm{MS} / \mathrm{KI} / \mathrm{CI}$ \\
\hline $\begin{array}{c}\text { (E,E)-Cosmene } \\
(15.875,1132)\end{array}$ & nd & nd & nd & nd & nd & $\begin{array}{c}0.4 \\
(0.2)\end{array}$ & $\begin{array}{c}0.6 \\
(0.2)\end{array}$ & nd & $\mathrm{MS} / \mathrm{KI}$ \\
\hline $\begin{array}{c}\text { 6,10-Dimethyl-5,9-undecadien-2-one } \\
(28.883,1453)\end{array}$ & $\begin{array}{c}0.7 \\
(0.0)\end{array}$ & $\begin{array}{c}0.6 \\
(0.4)\end{array}$ & nd & $\begin{array}{c}0.7 \\
(0.0)\end{array}$ & nd & $\begin{array}{c}0.7 \\
(0.4)\end{array}$ & $\begin{array}{l}1.0 \\
(0.2)\end{array}$ & $\begin{array}{c}0.6 \\
(0.2)\end{array}$ & $\mathrm{MS} / \mathrm{KI}$ \\
\hline $\begin{array}{c}\text { Isocaryophyllene } \\
(30.511,1409)\end{array}$ & nd & nd & nd & nd & nd & $\begin{array}{c}0.3 \\
(0.0)\end{array}$ & $\begin{array}{c}0.2 \\
(0.0)\end{array}$ & $\begin{array}{l}0.4 \\
(0.0)\end{array}$ & MS/KI \\
\hline $\begin{array}{l}\text { (E)-Nerolidol } \\
(33.432,1564)\end{array}$ & nd & $\begin{array}{c}0.3 \\
(0.0)\end{array}$ & nd & $\begin{array}{c}0.2 \\
(0.0)\end{array}$ & nd & nd & nd & $\begin{array}{c}0.2 \\
(0.0)\end{array}$ & $\mathrm{MS} / \mathrm{KI}$ \\
\hline $\begin{array}{c}\text { (-)-Spathulenol } \\
(33.959,1566)\end{array}$ & $\begin{array}{c}0.2 \\
(0.0)\end{array}$ & $\begin{array}{c}0.3 \\
(0.0)\end{array}$ & nd & $\begin{array}{c}0.2 \\
(0.0)\end{array}$ & $\begin{array}{c}0.3 \\
(0.0)\end{array}$ & nd & $\begin{array}{c}0.2 \\
(0.0)\end{array}$ & nd & $\mathrm{MS} / \mathrm{KI}$ \\
\hline $\begin{array}{l}\text { 3,4-Dimethyl-3-cyclohexen-1-carboxaldehyde } \\
\qquad(34.520,1492)\end{array}$ & nd & nd & nd & nd & $\begin{array}{c}3.4 \\
(1.6)\end{array}$ & nd & nd & nd & \\
\hline $\begin{array}{l}\text { Cis-p-metha-1(7)-8-dien-2-ol } \\
(35.208,1233)\end{array}$ & nd & nd & nd & nd & nd & nd & nd & $\begin{array}{c}1.1 \\
(0.0)\end{array}$ & $\mathrm{MS} / \mathrm{KI}$ \\
\hline $\begin{array}{c}\text { Isoaromadendrene epoxide } \\
(37.465,1577)\end{array}$ & nd & nd & nd & $\begin{array}{c}0.8 \\
(0.2)\end{array}$ & nd & $\begin{array}{c}0.3 \\
(0.0)\end{array}$ & nd & nd & $\mathrm{MS} / \mathrm{KI}$ \\
\hline $\begin{array}{l}\text { 1,4-Dihydroxy-p-menth-2-ene } \\
(37.767,1243)\end{array}$ & $\begin{array}{c}1.1 \\
(0.0)\end{array}$ & $\begin{array}{c}2.3 \\
(2.1)\end{array}$ & nd & nd & nd & nd & $\begin{array}{l}1.3 \\
(0.4)\end{array}$ & nd & $\mathrm{MS} / \mathrm{KI}$ \\
\hline $\begin{array}{c}\beta \text {-Farnesene } \\
(39.188,1456)\end{array}$ & nd & nd & nd & nd & nd & nd & nd & $\begin{array}{c}0.8 \\
(0.5)\end{array}$ & $\mathrm{MS} / \mathrm{KI}$ \\
\hline $\begin{array}{c}\text { Farnesol(E)-methylether } \\
(39.186,1682)\end{array}$ & $\begin{array}{c}0.8 \\
(0.0)\end{array}$ & nd & nd & nd & nd & nd & nd & $\begin{array}{c}0.2 \\
(0.0)\end{array}$ & $\mathrm{MS} / \mathrm{KI}$ \\
\hline Farnesol & nd & $\begin{array}{c}6.3 \\
(1.3)\end{array}$ & nd & $\begin{array}{c}4.5 \\
(2.1)\end{array}$ & $\begin{array}{c}5.9 \\
(1.7)\end{array}$ & nd & $\begin{array}{c}1.2 \\
(0.8)\end{array}$ & $\begin{array}{c}0.2 \\
(0.0)\end{array}$ & $\mathrm{MS} / \mathrm{KI} / \mathrm{CI}$ \\
\hline $\begin{array}{c}\beta \text {-Springene } \\
(39.410,1918)\end{array}$ & nd & $\begin{array}{c}0.2 \\
(0.0)\end{array}$ & $\begin{array}{c}0.2 \\
(0.1)\end{array}$ & $\begin{array}{c}0.2 \\
(0.0)\end{array}$ & & $\begin{array}{c}2.0 \\
(0.3)\end{array}$ & $\begin{array}{c}0.9 \\
(0.1)\end{array}$ & $\begin{array}{l}2.0 \\
(0.1)\end{array}$ & $\mathrm{MS} / \mathrm{KI}$ \\
\hline
\end{tabular}


Table 3. Cont.

\begin{tabular}{|c|c|c|c|c|c|c|c|c|c|}
\hline $\begin{array}{c}\text { Compounds } \\
(\mathrm{RT}, \mathrm{KI})^{1}\end{array}$ & $\begin{array}{c}\text { TV1 } \\
\text { muya }\end{array}$ & $\begin{array}{c}\text { TV2 } \\
\text { muya }\end{array}$ & $\begin{array}{c}\text { TV3 } \\
\text { muya }\end{array}$ & $\begin{array}{c}\text { TV3 } \\
\text { muyb }\end{array}$ & $\begin{array}{l}\text { TV3 } \\
\text { muyc }\end{array}$ & $\begin{array}{c}\text { TV4 } \\
\text { muya }\end{array}$ & $\begin{array}{c}\text { TV4 } \\
\text { muyb }\end{array}$ & $\begin{array}{c}\text { Tv4 } \\
\text { muyc }\end{array}$ & $\begin{array}{c}\text { Identification } \\
\text { Method }^{2}\end{array}$ \\
\hline $\begin{array}{c}\alpha \text {-Springene } \\
(40.793,1731)\end{array}$ & nd & nd & nd & nd & $\begin{array}{c}0.2 \\
(0.0)\end{array}$ & nd & nd & $\begin{array}{c}0.2 \\
(0.0)\end{array}$ & $\mathrm{MS} / \mathrm{KI}$ \\
\hline $\begin{array}{l}\text { Hexadecane } \\
(42.274,1818)\end{array}$ & $\begin{array}{c}0.2 \\
(0.0)\end{array}$ & $\begin{array}{c}0.5 \\
(0.1)\end{array}$ & nd & $\begin{array}{c}0.3 \\
(0.1)\end{array}$ & nd & nd & nd & nd & \\
\hline Density & $\begin{array}{c}0.988 \\
(0.038)\end{array}$ & $\begin{array}{c}0.988 \\
(0.012)\end{array}$ & $\begin{array}{c}0.942 \\
(0.001)\end{array}$ & $\begin{array}{c}0.973 \\
(0.000)\end{array}$ & $\begin{array}{c}0.973 \\
(0.027)\end{array}$ & $\begin{array}{c}1.030 \\
(0.030)\end{array}$ & $\begin{array}{c}1.000 \\
(0.000)\end{array}$ & $\begin{array}{r}1.017 \\
(0.017)\end{array}$ & \\
\hline Yield $(\% w / w)$ & $\begin{array}{c}0.20 \\
(0.01)\end{array}$ & $\begin{array}{c}0.22 \\
(0.01)\end{array}$ & $\begin{array}{l}0.16 \\
(0.00)\end{array}$ & $\begin{array}{c}0.18 \\
(0.00)\end{array}$ & $\begin{array}{c}0.18 \\
(0.00)\end{array}$ & $\begin{array}{c}0.18 \\
(0.00)\end{array}$ & $\begin{array}{c}0.17 \\
(0.01)\end{array}$ & $\begin{array}{c}0.18 \\
(0.01)\end{array}$ & \\
\hline
\end{tabular}

Data presented as mean (SEM)/density (SEM) ${ }^{1} \mathrm{RT}=$ Retention time and $\mathrm{KI}=$ Kovats index relative to Zebron ZB-5 column. KI is compared with [18] $(90 \% \mathrm{KI}) .{ }^{2} \mathrm{M}=\mathrm{mass}$ spectrum matching with NIST library [17], CI = co-injection with standard. 
The densities of these oils were between $0.959 \pm 0.013 \mathrm{~g} / \mathrm{mL}$ and $1.087 \pm 0.016 \mathrm{~g} / \mathrm{mL}$ for oils extracted from Kyadongo B samples and for Muyago samples, it varied between $0.942 \pm 0.001 \mathrm{~g} / \mathrm{mL}$ and $1.030 \pm 0.030 \mathrm{~g} / \mathrm{mL}$. The compositions were expressed as mean peak areas (\% $\pm \mathrm{SEM})$ of the major compounds quantified from the sample sites of the two study areas. The highest composition representation came from the alkylbenzenes (ethylbenzene, o-xylene, $\mathrm{p}$-xylene and $\mathrm{m}$-xylene). Among these, the composition of o-xylene was highest and varied between $n . d-29.4 \pm 1.6 \%$ in samples from Kyadong B village and nd $-23.4 \pm 17.3 \%$ for Muyago village. This was closely followed by $\mathrm{m}$-xylene with nd $-25.0 \pm 1.2 \%$ (Kyadongho B village) and nd-18.1 $\pm 2.7 \%$ (Muyago village) and finally ethylbenzene with nd $-4.0 \pm 0.4 \%$ (Kyadong B) and nd $-2.7 \pm 0.2 \%$ (Muyago) in this category. There was a significant amount of farnesol, varying between nd- $6.3 \pm 1.3 \%$ (Muyango) and nd $-2.9 \pm 0.2 \%$ (Kyadongho B). $\beta$-Springene was another major compound with the composition between nd-2.0 $\pm 0.3 \%$ (Muyago samples) and nd $-5.7 \pm 2.9 \%$ (Kyadongho B samples).

\subsection{Chemotypes in T. vogelii Essential Oil}

To determine the correlation of major components between various T. vogelii samples, PCA and AHC were performed on the data. Principal component analysis led to a total of eight factors extracted (i.e., whose eigenvalues were greater than unity) and the loading scores were generated. These components could explain about $88 \%$ of the total variance. Multiple linear regression (MLR) of the elements in the factor score matrix was carried out against the total composition for the data to estimate the contribution of each major component in the chemotype on total component composition of the samples. Significance of the regression coefficients $\left(R^{2}=0.95\right.$, observations; $\left.N=19\right)$ was at $95 \%$ confidence level (CI) $(p<0.05)$. The regression results based on eight factor scores showed that components four $(p=0.35)$, five $(p=0.63)$, six $(p=0.25)$, seven $(p=0.96)$ and eight $(p=0.33)$ did not significantly influence component composition. Therefore, the components were reduced to three factors, which explained $55 \%$ of the total variance. PC1 could describe about $30 \%$, PC2 could describe about $14 \%$, and PC 3 could describe $11 \%$ of the total variance. The MLR equation $\left(R^{2}=0.95\right.$, ANOVA significance $\mathrm{f}<23.43, p<0.05)$ was as follows: total composition $=19.39 \mathrm{SC} 1+7.70 \mathrm{SC} 2+1.92 \mathrm{SC} 4+$ 19.4, where SC1, SC2 and SC4 are factor scores for samples in components 1, 2 and 3, respectively.

Graphical representation of the principal components (Figure 1) reveal three chemical groupings. The first category shows that some samples were majorly farnesol (Fnso) type (chemotype 1). Farnesol was detected in the following samples: TV1kyb, TV1kyc, TV1 muya, TV2muya, TV2kyd, TV3muyc, TV4kya, TV4kyb and TV4kyc, where in some cases it was one of the major components. Among other compounds in this grouping were 2-butoxyethanol (E2B) and D-(+)-Alpha-pinene $(\alpha \mathrm{P})$. Not all these other compounds could be detected in each of these samples (as was the case for farnesol). The second grouping was majorly springene compounds ( $\beta$-springene ( $\beta S$ ) and $\alpha$-springene $(\alpha \operatorname{Sp})$ ) and $\beta$-farnesene $(\beta F)$, which was referred to as chemotype 2 . There is a positive correlation between $\beta$-springene and $\beta$-farnesene (Pearson correlation, $r=0.3$ ), and $\alpha$-springene and $\beta$-farnesene (Pearson correlation $r=0.2$ ). However, there is no positive correlation between either $\beta$-springene or $\beta$-farnesene with farnesol, signifying a different chemical grouping. $\beta$-springene, $\alpha$-springene and $\beta$-farnesene were detected in the rest of the samples other than those detected for farnesol above (TV3muyb, TV3muya, TV2kyb, TV2kya TV4muya and TV4muyc, TV2kyc and TV1kya). The other compounds in chemotype 2 were: (E)-nerolidol (EN), cis-p-metha-1(7)-8-dien-2-ol (zMD), D-limonene (Dl), 1,4-dihydroxy-p-menth-2-ene (Dm) 6,10-dimethyl-5,9-undecadien-2-one (UD), and farnesol (E)-methylether (FnsoEm). $\beta$-Springene was the most represented in this category since it was encountered in six samples out of a total of 19 samples (TV3muyb, TV3muya, TV2kyb, TV2kya TV4muya and TV4muyc). $\beta$-Farnesene was detected in two samples (TV1kya and TV2kyc). All samples, however, were dominated by the alkylbenzenes: ethylbenzenes and xylene isomers either in abundance or trace amounts (treated as non-detectable). There was minimum composition of the mixture of farnesene compounds (farnesol, $\beta$-springene, $\alpha$-springene and $\beta$-farnesene) with a huge amount of alkylbenzenes, which formed the mixed chemotype (i.e., in samples like TV4muyb and TV3kya). There is a large correlation between 
alkylbenzenes and farnesol (Pearson correlation, $\mathrm{r}>0.4, p<0.05$, for all alkylbenzenes) and also a correlation between $\alpha$-springene and farnesol (Pearson correlation, $\mathrm{r}>0.4, p<0.05$ ).

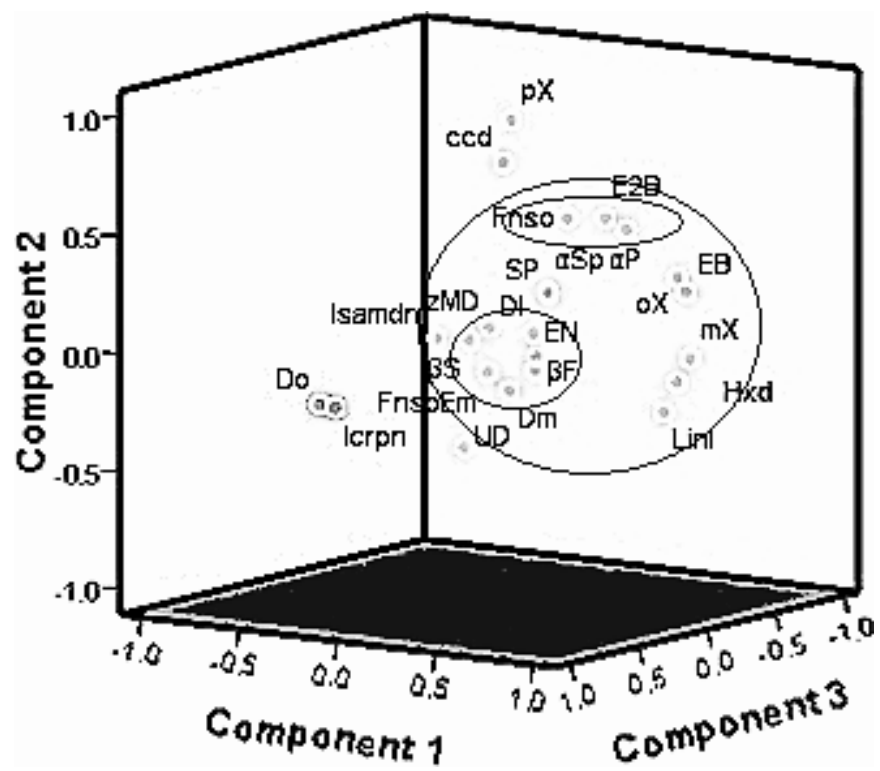

Figure 1. Three dimensional scatter plot of different correlations of chemical components using principal component analysis. Other acronyms: p-Xylene (pX), Linalool (Linl), Isocaryophyllene (Icrpn), (-)-Spathulenol (SP), (E,E)-Cosmene (Do), 3,4-dimethyl-3-cyclohexen-1-carboxaldehyde (ccd), Hexadecane (Hxd), Isoaromadendrene epoxide (Isamdn).

Figure 2 shows the major hierarchical clustering classification of major components in the oil. Farnesol, $\beta$-springene, $\alpha$-springene and $\beta$-farnesene could form separate clusters, affirming the above observations. A cluster of ethylbenzene (EB), o-xylene (oX) and m-xylene (mX) was formed. The hydrocarbon cluster was the first in the dendrogram. Thus, the compounds within this cluster are broadly similar to each other. These arguments are in line with those suggested by [10] who obtained three chemotypes from T. vogelii samples from east Africa (Kenya, Tanzania and Malawi) using phytochemical analysis. Chemotype 1 had rotenoids, chemotype 2 lacked rotenoids but had flavanones and flavones, while chemotype 3 had a hybrid chemical profile of chemotype 1 and chemotype 2 .

\subsection{Effect of Season Variation on the Percentage Yield and Major Composition of the Oils}

Two seasonal variations were considered in the study: the rainy season and the dry season are represented with the green pattern and red pattern, respectively (Figure 3). The rainfall bimodal peaks in the district occur between March to May and August to September. Samples obtained during this time were: TV1muya, TV2muya, TV3muya, TV3muyb, TV3muyc, TV2kya, TV2kyb, TV2kyc, TV2kyd, and TV3kya. During the dry season, harvest occurred in January and June and these samples were: TV1kya, TV1kyb, TV1kyc, TV4kya, TV4kyb, TV4kyc, TV4muya, TV4muyb, and TV4muyc. Considering this, there was no significant difference between the percentage oil yield between the two seasons and from the two sampled areas. 


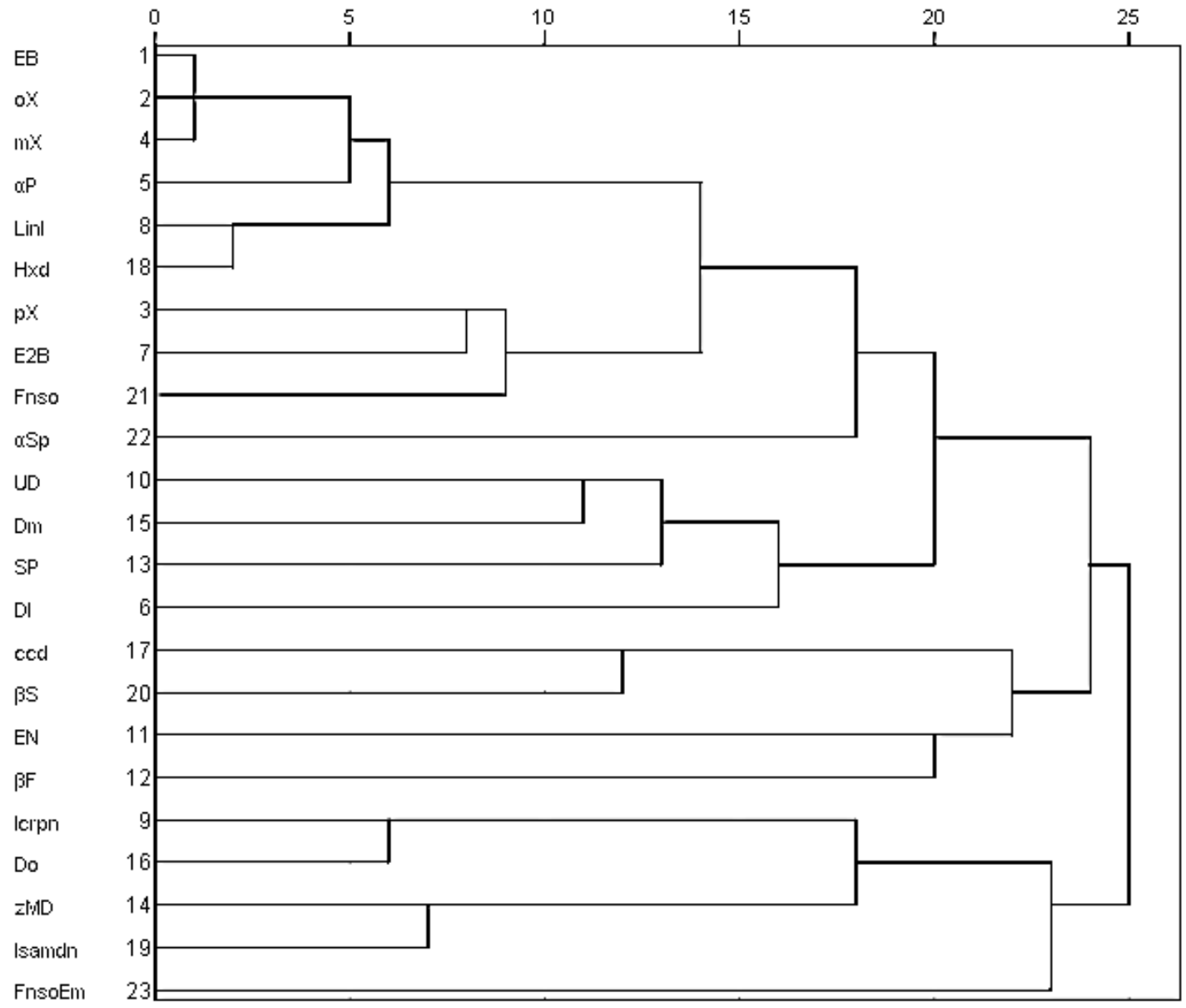

Figure 2. Dendrogram of major components obtained based on the classification of the samples of $T$. vogelii during the two harvest periods.

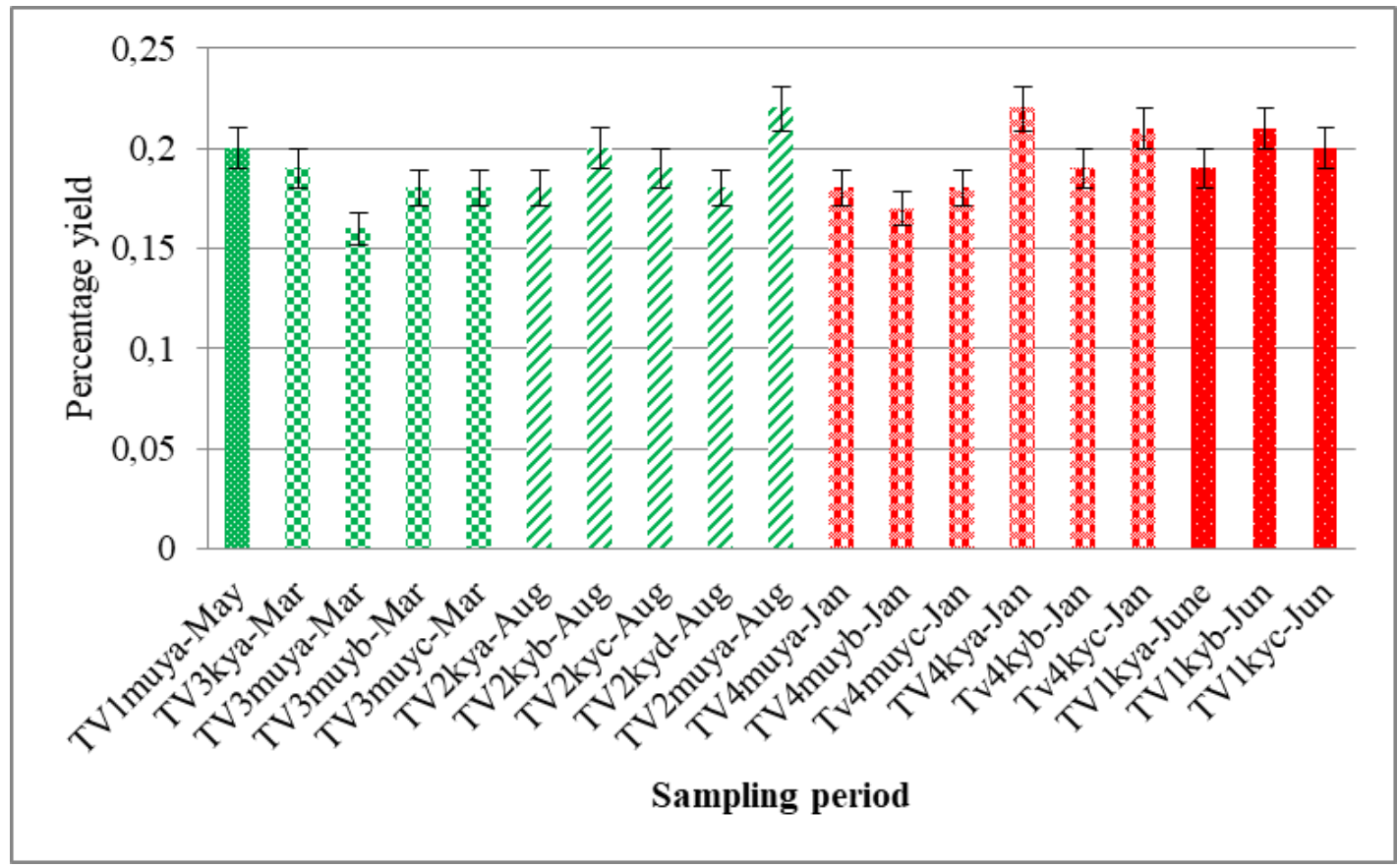

Figure 3. A continuous histogram depicting the percentage yield of the oils from samples of T. vogelii. 
In Figure 4, cluster 1 mainly contained the composition of the samples taken during the rainy season in March-May and August (TV1muya, TV2muya, TV3kya) and one sample from the dry season in June (TV1 kyb). Clusters 2 and 4 were compositions for the samples during rainy season sampling, i.e., cluster 2-Tv3muyb and Tv3muyc were from March sampling, and cluster 4-TV2kya and TV2kyb from August sampling. In addition, Tv3muya that formed Cluster 6 was sampled in March (the rainy season). Clusters 3 and 5 were for major compounds of the samples from both dry and rainy seasons (TV1kyc and TV2kyd for cluster 3 and TV2kyc and TV1kya for cluster 5). Finally, samples in cluster 7 (TV4muya and TV4muyc), cluster 8 (TV4kyc and TV4muyb), cluster 9 (TV4kyb) and cluster 10 (TV4kya) were obtained during the dry season (January). These correlations indicate major seasonal effects on the composition of the major constituents. However, compounds like ethylbenzene, o-xylene and $\mathrm{m}$-xylene could not be detected in the samples that were picked in January, but were observed in samples for June. The compounds were, however, found in trace amounts and therefore not quantified. This clustering certainly could have serious implications on the pesticidal activity of the T. vogelii leaf material.

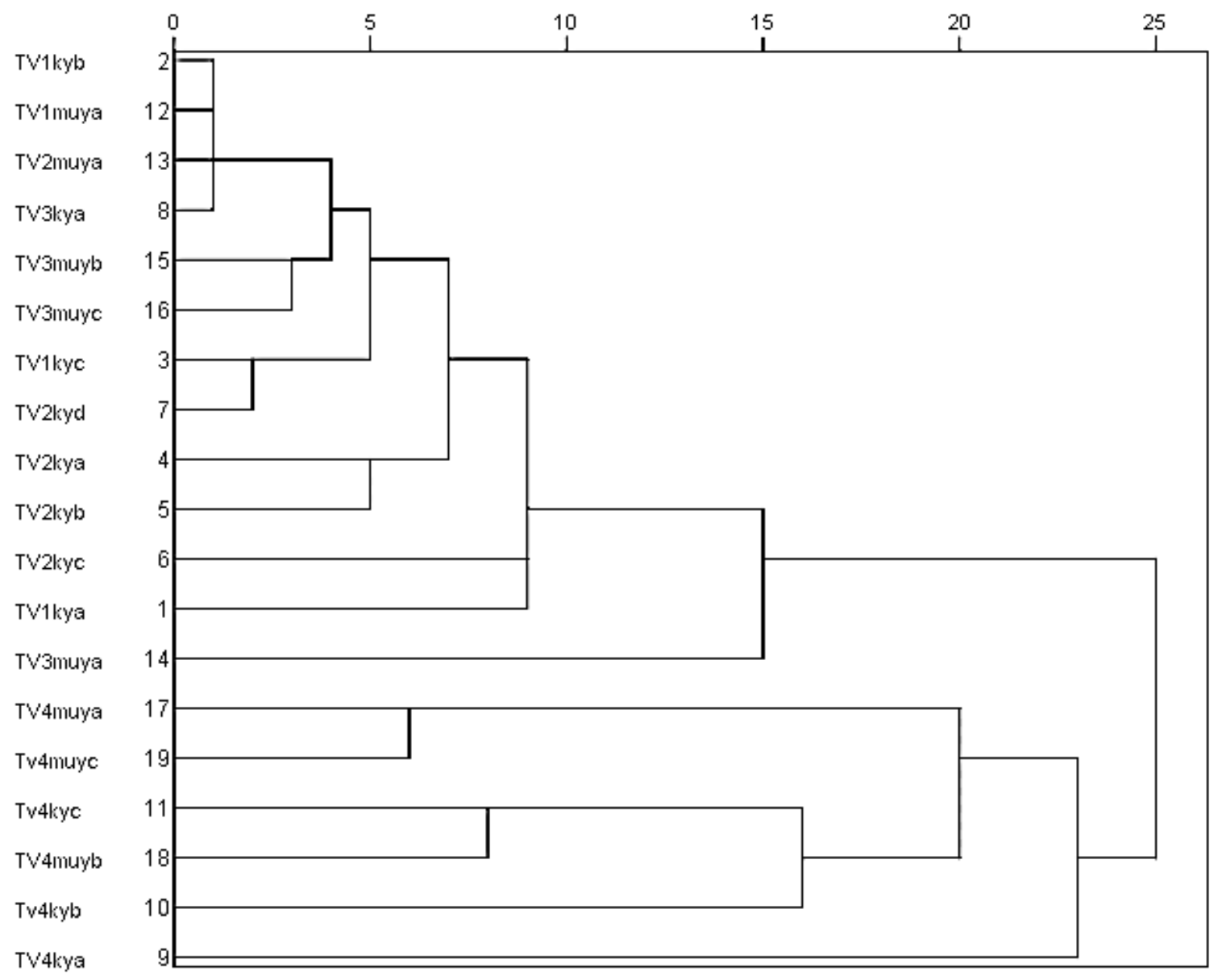

Figure 4. Dendrogram of samples due to classification based on their major composition.

\subsection{Chemotaxonomic Significance of These Chemical Varieties}

These observations reveal a very significant chemotaxonomic importance where in this part of the world, T. vogelii is of three chemical varieties: the farnesol variety, the springene variety, and the mixed variety. Figure 5 shows the farnesene compounds that formed the different chemotypes.

Farnesol is an oxygenated sesquiterpene of various isomers; $(E, Z)$-farnesol, $(Z, E)$-farnesol and $(E, E)$-farnesol. The most common isomer is $(E, E)$-farnesol, which represents $95 \%$ of all farnesol and was the most identified. Springene is an isoprenoid hydrocarbon of diterpene nature. The two 
springenes, $\beta$-springene (7,11,15-trimethyl-3-methylene-1,6,10,14-hexadecatetraene) and $\alpha$-springene (E,E,E-3,7,11,15-tetramethyl-1,3,6,10,14-hexadecapentaene), were identified. Both farnesol and the springenes, however, belong to the farnesene family. $(E, E)-\beta$-springene is a diterpene homolog of (E)- $\beta$-farnesene (4) (which formed part of the second grouping).

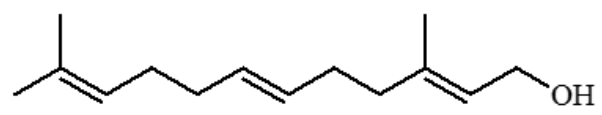

Farnesol

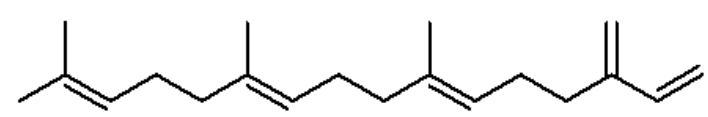

Beta springene

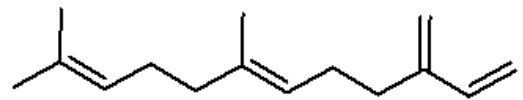

Beta -farnesene

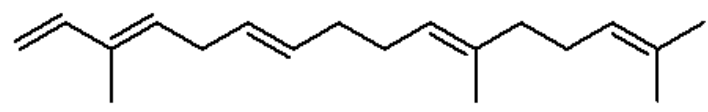

Alpha springene

Figure 5. Compounds of chemotaxonomic significance identified from T. vogelii.

These are quite uncommon compounds. However, $\beta$-springene has been previously found in the essential oils derived from the leaves of the herb Heracleum persicum Desf. ex Fischer from Kandavan, northern Tehran in Iran [21] and Sigesbeckia jorullensis Kunth (Asteraceae) from north-east of Hamburg, Germany [22]. It was also detected in Lagochilus cabulicus Benth (Lamiaceae) (19.4\%), an aromatic plant from the Wakhan Corridor in Afghanistan used by the Wakhi and Kyrgyz peoples [23]. Additionally, it was found in very small amounts in Salvia sclarea Clary (Lamiaceae) $(1.1 \%)$ from France [24] and Salvia reuterana Boiss (Lamiaceae) (0.3\%) from Iran [25]. $\alpha$-Springene was identified only in the essential oil of Murraya exotica L. (Rutaceae) flowers collected in India, where it was the major constituent (23.8\%) [26], and in the essential oil of Teucrium marum L. (Lamiaceae) from Corsica, where it was detected as one of the main compounds (1.1-17.8\%) [27]. (E)- $\beta$-Farnesene is also an aphid alarm pheromone; in a Y-tube olfactometer bioassay, female Aphidius uzbekistanicus Luzhetski were attracted to aphid groups under attack from parasitoids, acting as a host-finding kairomone [28]. High concentrations of (E)- $\beta$-farnesene are believed to be potentially important for agricultural pest management strategies, but its role as a kairomone in the field or natural settings has been doubted [29]. Farnesol exhibits fungicidal activity against Paracoccidioides brasiliensis. High farnesol concentrations reduced cadmium toxicity and antioxidant responses [30]. It is therefore noteworthy to report the presence of $\alpha$ - and $\beta$-springene as very important constituents of Ugandan T. vogelii essential oils.

\subsection{Evaluation of the Repellency Potential of the Chemotypes of the Volatile Constituents of T. vogelii}

The repellency potential of TV4kyc (farnesol chemotype), TV4muya (springene chemotype) and TV1kyb (mixed chemotype) was also evaluated and the results indicate that there was not much difference in the repellency effect of the farnesol and springene chemotypes against $S$. zeamais, unlike the mixed one (Figure 6). The preference index of TV4kyc (farnesol type) oil against $S$. zeamais ranged between 0.0 to $-0.7,0.0$ to -0.5 and -0.3 to -1 for $0.03 \mu \mathrm{L}$ of oil per $\mathrm{cm}^{3}$ of air, $0.16 \mu \mathrm{L}$ of oil per $\mathrm{cm}^{3}$ of air and $0.31 \mu \mathrm{L}$ of oil per $\mathrm{cm}^{3}$ of air, respectively. The preference index of TV4muya (springene type) could vary between 0.1 to $-0.4,-0.2$ to -0.5 and -0.4 to -1 for $0.03 \mu \mathrm{L}$ of oil per $\mathrm{cm}^{3}$ of air, $0.16 \mu \mathrm{L}$ of oil per $\mathrm{cm}^{3}$ of air and $0.31 \mu \mathrm{L}$ of oil per $\mathrm{cm}^{3}$ of air treatment. These results indicate that the oils had a repellency effect against the maize weevil based on the preference index scale of $S$. zeamais. Chemotype 3 (mixed) showed a lower effect. The composition of farnesol and springene was very low, partly explaining the lower repellency activity of this chemotype. These observations underline the vital role that both farnesol and springene play in the repellency potential of the essential oil derived from T. vogelii. 


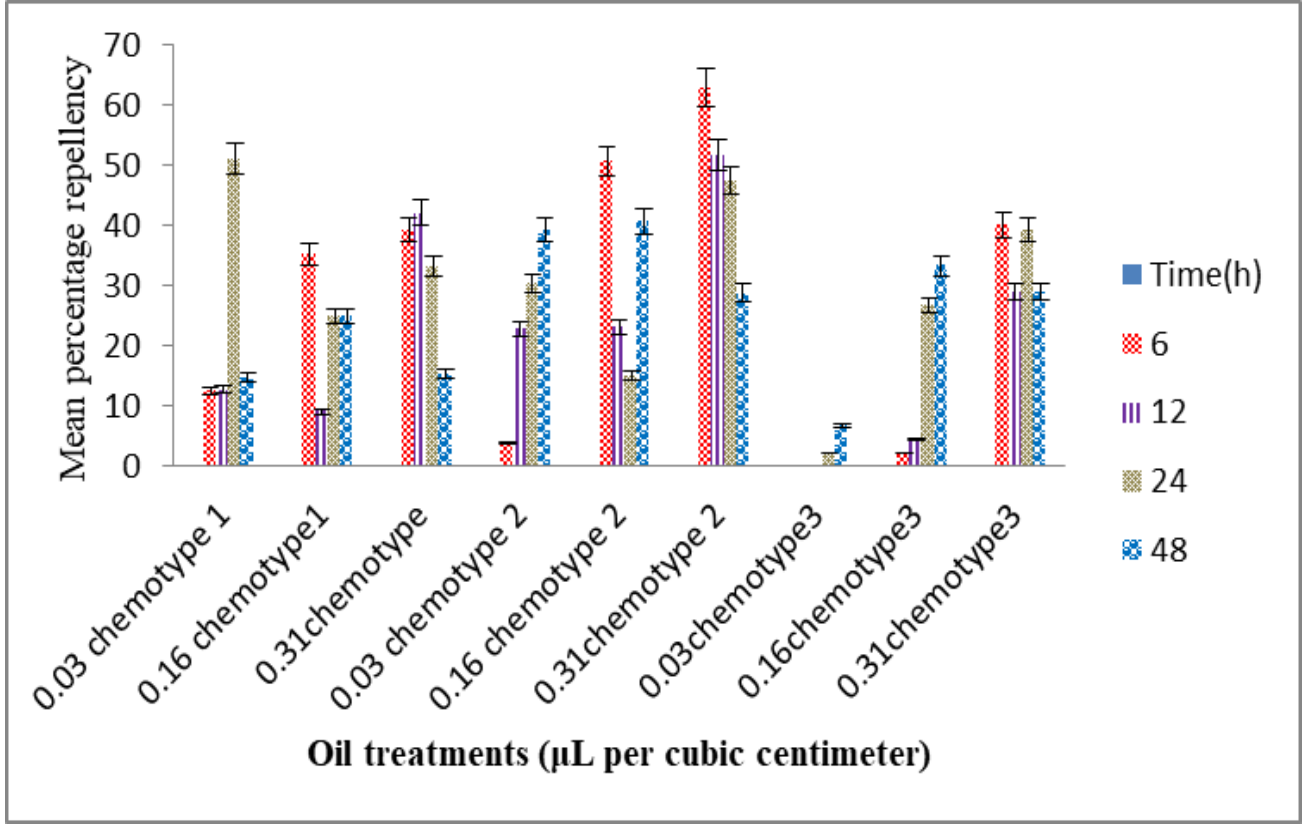

Figure 6. Mean percentage repellence of three varieties of T. vogelii against S. zeamais.

The repellency potential of farnesol was also evaluated against the weevil (as shown below in Figure 7) and results show that farnesol is effective at higher concentrations as a repellent against S. zeamais. The preference index evaluated for $0.31 \mu \mathrm{L}$ of farnesol per $\mathrm{cm}^{3}$ of air varied between 0.0 to -0.4 , while that of the lower concentration ranged between 0.1 to 0.6 and -0.2 to 0.3 for $0.16 \mu \mathrm{L}$ and $0.03 \mu \mathrm{L}$ of farnesol per $\mathrm{cm}^{3}$ of air, respectively. This implied that at lower concentrations, farnesol had a very limited repellent effect against $S$. zeamais. Repellency activity roughly increased with an increase in the amount of farnesol.

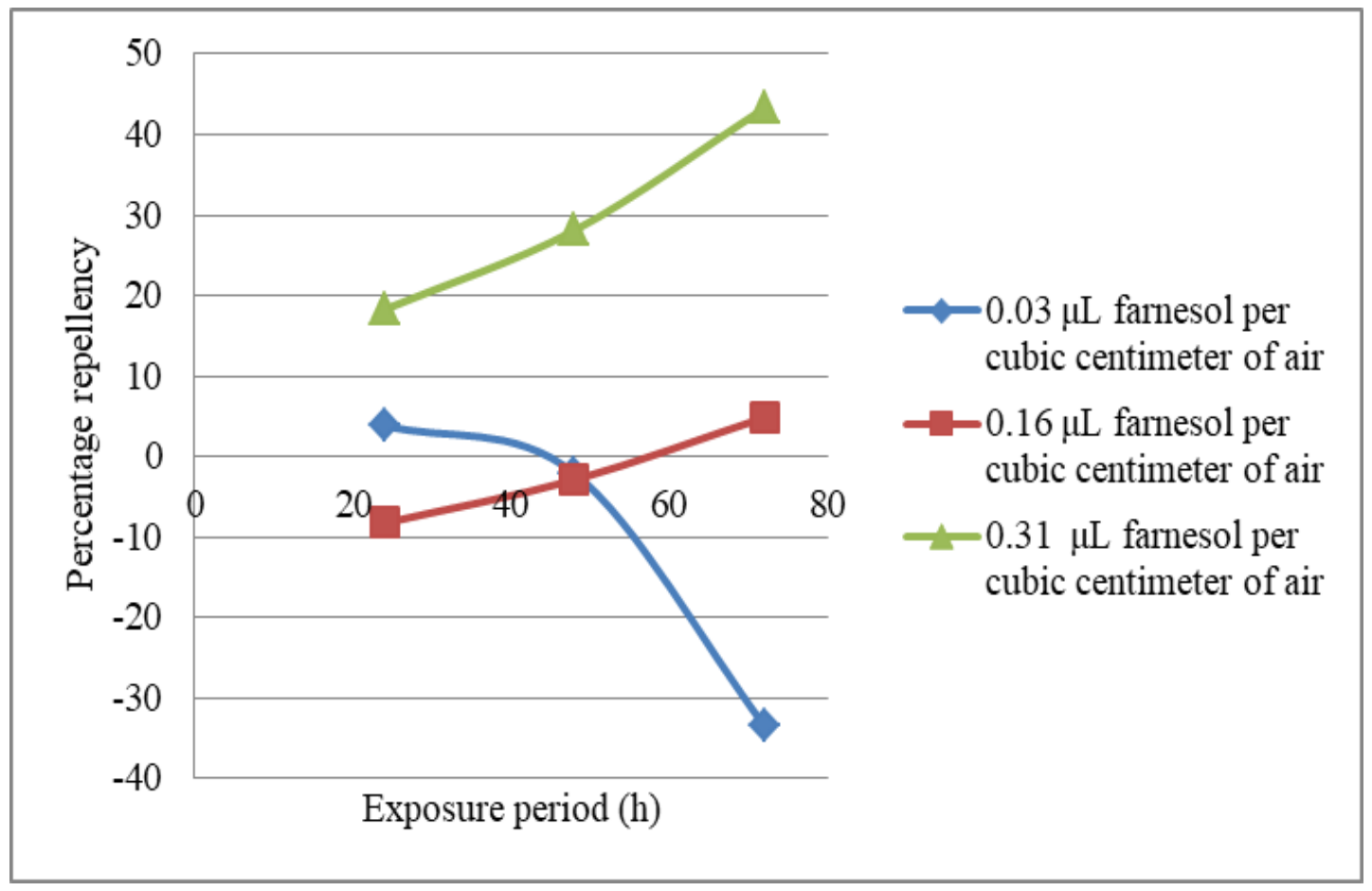

Figure 7. Mean percentage repellence of the farnesol standard against S. zeamais. 
The implication of this repellency effect of farnesol isomers is that the farnesol type could have it repellency activity increased with an increase in the amount of farnesol in the oil and probably would have an advantage over the springene type. In both cases, however, the synergistic or complementary part of all other compounds found in the same oils play a crucial role in the overall repellent and insecticidal effect of this oil. The chemical profiles of this T. vogelii chemotype in essential oils almost resembles those of Lippia javanica essential oils. The only difference is that the L. javanica chemotypes had one of its major components shown to be greatly inactive [9]. In both cases, there was a large variation in the composition of their major components. The yields, however, varied only for the L. javanica chemotypes, but not for the chemotypes of the essential oils of T. vogelii in this study.

The overall percentage repellency was relatively small, thus giving a short protection time. A repellence of nearly $100 \%$ at a relatively long time would be preferred. The short protection time could be improved via formulation technology development through retaining the active ingredient on the skin of the insects for longer periods. This can be made possible by preparing cream-based and polymer mixture-based formulations.

\subsection{Conclusions}

The investigation of the chemical varieties present in the essential oils of $T$. vogelii species from the eastern part of Uganda has revealed three chemotypes: either farnesol, or the springene and $\beta$-farnesene type, or one with a mixed chemical profile of the two chemotypes based on the compounds of farnesene family (chemotype three). Geographical and seasonal variation did not affect the amount of the oil significantly; however, the composition and the constituents of the oils were affected by the harvest period. Evaluation of the repellency effects of these chemical varieties of T. vogelii showed that chemotypes 1 and 2 were similarly active but more repellent than chemotype 3 against $S$. zeamais. At a lower concentration, there was no significant repellency effect between chemotypes 1 and 2 . At higher concentrations, the effect could change. Notwithstanding, the repellent effect of these varieties of T. vogelii still holds. The complementary part of all other compounds found in the same oils plays a crucial role in the overall repellent effect of this oil. However, more study is needed that aims to optimize and standardize the chemical varieties and harvesting period needed for recommendation to smallhold farmers (especially under field conditions) before it can be adopted more widely. In addition, toxicity assays should be designed to identify the insecticidal properties of these chemotypes, including other repellency assay designs different from the petri dish assay design used here. This is because the petri dish is very small and the weevils are left in it for a very long time. Within minutes, the air in the petri dish could saturate and the choice will essentially be between a slightly lower and slightly higher concentration of the test compound. Within hours, the two sides may be rather indistinguishable.

Supplementary Materials: The following are available online at http://www.mdpi.com/2077-0472/10/5/164/s1. Figure S1: GC- Chromatogram for the standard mixture of compounds; Table S1: Loading score of extracted principal components; Table S2: Pearson correlation table of major components.

Author Contributions: Conceptualization, N.K. and S.K.K.; methodology, N.K. and S.K.K.; software, N.K. and A.O.O.; validation, A.O.O., R.B., O.O.O. and S.K.K.; formal analysis, N.K.; investigation, N.K.; resources, A.O.O. and O.O.O.; data curation, N.K.; writing — original draft preparation, N.K.; writing - review and editing, N.K. and S.K.K.; visualization, N.K.; supervision, A.O.O., O.O.O., R.B. and S.K.K.; project administration, O.O.O.; funding acquisition, A.O.O. and O.O.O. All authors have read and agreed to the published version of the manuscript.

Funding: This research received funding from the National Research Foundation-The World Academy of Sciences (NRF-TWAS) (grant number: 105468), University of Fort Hare, Govan Mbeki Research and Development Centre (GMRDC) and Directorate of Research and Development Walter Sisulu University and The APC was funded by University of Fort Hare.

Acknowledgments: We gratefully acknowledge funding from the National Research Foundation-The World Academy of Sciences (NRF-TWAS) (grant number: 105468), University of Fort Hare, Govan Mbeki Research and Development Centre (GMRDC) and Directorate of Research and Development Walter Sisulu University.

Conflicts of Interest: The authors declare no conflict of interest. The funders had no role in the design of the study; in the collection, analyses, or interpretation of data; in the writing of the manuscript, or in the decision to publish the results. 


\section{References}

1. Matovu, H.; Olila, D. Acaricidal activity of Tephrosia vogelii extracts on nymph and adult ticks. Int. J. Trop Dis. 2007, 2, 83-88.

2. Neuwinger, H.D. Plants used for poison fishing in tropical Africa. Toxicon 2004, 44, 417-430. [CrossRef] [PubMed]

3. Mwaura, L.; Anjarwalla, P.; Ofori, D.A.; Stevenson, P.C.; Smith, P.; Jamnadass, R. PesticidaL Plant. Leaflet. The Agroforestree Database-World Agroforestry. 2015. Available online: www.worldagroforestry.org (accessed on 18 February 2020).

4. Kamanula, J.F.; Sileshi, G.; Belmain, S.R.; Sola, P.; Mvumi, B.; Nyirenda, G.K.C.; Nyirenda, S.P.; Stevenson, P.C. Farmers' insect pest management practices and pesticidal plant use in the protection of stored maize and beans in southern Africa. Int. J. Pest. Manag. 2011, 57, 41-49.

5. Nyirenda, S.P.; Sileshi, G.; Belmain, S.R.; Kamanula, J.F.; Mvumi, B.; Sola, P.; Nyirenda, G.K.C.; Stevenson, P.C. Farmers' ethno-ecological knowledge of vegetable pests and their management using pesticidal plants in northern Malawi and eastern Zambia. Afr. J. Agric. Res. 2011, 6, 1525-1537.

6. $\quad$ Ogendo, J.O.; Belmain, S.R.; Deng, A.L.; Walker, D.J. Efficacy of Lantana camara L. and Tephrosia vogelii Hook against Sitophilus zeamais (Coleoptera: Curculionidae) in stored maize grains. In Proceedings of the III WOCMAP Congress on Medicinal and Aromatic Plants, Chiang Mai, Thailand, 3 February 2003; pp. $137-143$.

7. Koona, P.; Malaa, D.; Koona, O.E. Hexane extracts from Tephrosia vogelii Hook. f. protect stored maize against the weevil Sitophilus zeamais Motschulsky (Coleoptera: Curculionidae). Entomol. Sci. 2007, 10, 107-111. [CrossRef]

8. Stevenson, P.C.; Kite, G.C.; Lewis, G.P. Distinct Chemotypes of Tephrosia Vogelii and Implications for Their Use in Pest Control and Soil Enrichment. Phytochemistry 2012, 78, 135-146. [CrossRef]

9. Kamanula, J.F.; Belmain, S.R.; Hall, D.R.; Farman, D.I.; Goyder, D.J.; Mvumi, B.M.; Masumbu, F.F.; Stevenson, P.C. Chemical variation and insecticidal activity of Lippia javanica (Burm. f.) Spreng essential oil against Sitophilus zeamais Motschulsky. Ind. Crops. Prod. 2017. [CrossRef]

10. Mkindi, A.G.; Tembo, Y.; Mbega, E.R.; Medvecky, B.; Kendal-Smith, A.; Farrell, I.W.; Ndakidemi, P.A.; Belmain, S.R.; Stevenson, P.C. Phytochemical Analysis of Tephrosia vogelii across East Africa Reveals Three Chemotypes that Influence Its Use as a Pesticidal Plant. Plants 2019, 8, 597. [CrossRef]

11. Bendera, M.M. Isolation and Characterization of Essential Oils from Ocimum americanum, Lantana camara, Lantana trifolia and Tephrosia vogelii. Ph.D. Thesis, Egerton University, Njoro, Kenya, 2007.

12. Noudogbessi, J.P.; Sessou, P.; Wotto, V.D.; Figueredo, G.; Chalard, P.; Chalchat, J.C.; Sohounhloué, D.C.K. Chemical compositions and preventive activity of essential oils extracted from the leaves of two varieties of Tephrosia(Leguminosae-Papilionoideae) collected in Benin on Callosobruchus maculatus (Fabricius). AJRC 2012, 5, 1431-1436.

13. Lee, B.H.; Choi, W.S.; Lee, S.E.; Park, B.S. Fumigant toxicity of essential oils and their constituent compounds towards the rice weevil, Sitophilus oryzae (L.). Crop. Prot. 2001, 20, 317-320. [CrossRef]

14. Chaubey, M.K. Fumigant toxicity of essential oils and pure compounds against Sitophilus oryzae L. (Coleoptera: Curculionidae). Biol. Agric. Hortic. 2012, 28, 111-119. [CrossRef]

15. Government of Uganda. Atlas of Uganda; Government Printers: Entebbe, Uganda, 1967.

16. Oonyu, J. Upland rice growing: A potential solution to declining crop yields and the degradation of the Doho wetlands, Butaleja district-Uganda. Afr. J. Agric. Res. 2011, 6, 2774-2783.

17. Wannes, W.A.; Mhamdi, B.; Marzouk, B. Variations in essential oil and fatty acid composition during Myrtus communis var. italica fruit maturation. Food Chem. 2009, 112, 621-626. [CrossRef]

18. Babushok, V.I.; Linstrom, P.J.; Zenkevich, I.G. Retention Indices for Frequently Reported Compounds of Plant Essential Oils. J. Phys. Chem. Ref. Data 2011, 40, 043101. [CrossRef]

19. Johnson, G.W.; Ehrlich, R.; Full, W.; Ramos, S. Chapter 7-Principal Components Analysis and Receptor Models in Environmental Forensics. In Introduction to Environmental Forensics, 2nd ed.; Murphy, B.L., Morrison, R.D., Eds.; Academic Press: Burlington, ON, Canada, 2007; pp. 207-208.

20. Tapondjou, A.; Adler, C.; Fontem, D.; Bouda, H.; Reichmuth, C. Bioactivities of cymol and essential oils of Cupressus sempervirens and Eucalyptus saligna against Sitophilus zeamais Motschulsky and Tribolium confusum du Val. J. Stored Prod. Res. 2005, 41, 91-102. [CrossRef] 
21. Mojab, F.; Rustaiyan, A.; Jasbi, A.R. Essential oils of Heracleum persicum Desf. ex Fischer leaves. Daru 2002, 10,6-8.

22. Heinrich, G.; Pfeifhofer, H.W.; Stabentheiner, E.; Sawidis, T. Glandular Hairs of Sigesbeckia jorullensis Kunth (Asteraceae): Morphology, Histochemistry and Composition of Essential Oil. Ann. Bot. 2002, 89, 459-469. [CrossRef]

23. Jeppesen, A.S.; Soelberg, J.; Jager, A.K. Chemical composition of the essential oil from nine medicinal plants of the Wakhan Corridor, Afghanistan. J. Essent. Oil Bear. Plants 2012, 15, 204-212. [CrossRef]

24. Laville, R.; Castel, C.; Filippi, J.J.; Delbecque, C.; Audran, A.; Garry, P.P.; Legendre, L.; Fernandez, X. Amphilectane diterpenes from Salvia sclarea: Biosynthetic considerations. J. Nat. Prod. 2012, 75, 121-126. [CrossRef]

25. Karamian, R.; Asadbegy, M.; Pakzad, R. Essential oil compositions and in vitro antioxidant and antibacterial activities of the methanol extracts of two Salvia species (Lamiaceae) from Iran. Int. J. Agric. Crop Sci. 2013, 5, 1171-1182.

26. Raina, V.K.; Verma, S.C.; Dhawan, S.; Khan, M.; Ramesh, S.; Singh, S.C.; Yadav, A.; Srivastava, S.K. Essential oil composition of Murraya exotica from the plains of northern India. Flavour Fragr. J. 2006, 21, 140-142. [CrossRef]

27. Djabou, N.; Andreani, S.; Varesi, L.; Tomi, F.; Costa, J.; Muselli, A. Analysis of the volatile fraction of Teucrium marum L. Flavour Fragr. J. 2013, 28, 14-24. [CrossRef]

28. Micha, S.G.; Wyss, U. Aphid alarm pheromone (E)- $\beta$-farnesene: A host finding kairomone for the aphid primary parasitoid Aphidius uzbekistanicus (Hymenoptera: Aphidiinae). Chemoecology 1996, 7, 132-139. [CrossRef]

29. Joachim, C.; Weisser, W.W. Does the Aphid Alarm Pheromone (E)- $\beta$-farnesene Act as a Kairomone under Field Conditions. J. Chem. Ecol. 2015, 41, 267-275. [CrossRef] [PubMed]

30. Nunes, T.; Cardoso, P.; Freitas, R.; Figueira, E. Protective effects of farnesol on a Rhizobium strain exposed to cadmium. Ecotox. Environ. Safe 2018, 165, 622-629. [CrossRef]

(C) 2020 by the authors. Licensee MDPI, Basel, Switzerland. This article is an open access article distributed under the terms and conditions of the Creative Commons Attribution (CC BY) license (http://creativecommons.org/licenses/by/4.0/). 